\title{
5G Position and Orientation Estimation through Millimeter Wave MIMO
}

\author{
Arash Shahmansoori ${ }^{*}$, Gabriel E. Garcia ${ }^{\dagger}$, Giuseppe Destino ${ }^{\dagger \dagger}$, Gonzalo Seco-Granados* ${ }^{*}$, and Henk Wymeersch ${ }^{\dagger}$ \\ *Universitat Autònoma de Barcelona, Spain, Email: \{arash.shahmansoori, gonzalo.seco\}@uab.cat \\ ${ }^{\dagger}$ Chalmers University of Technology, Sweden, Email: \{ggarcia, henkw\}@ chalmers.se \\ ${ }^{\dagger}$ University of Oulu, Finland, Email: destino@ee.oulu.fi
}

\begin{abstract}
Millimeter wave and massive MIMO are considered enabling technologies for future 5G networks. While their benefits for achieving high-data rate communications are wellknown, their potential advantages for accurate positioning are largely undiscovered. We derive sufficient conditions under which transmission from a single mm-wave base station leads to a nonsingular Fisher information matrix associated with the position and orientation of a user terminal equipped with multiple antennas, which is in turn a prerequisite for joint estimation of the position and orientation.
\end{abstract}

\section{INTRODUCTION}

5G communication networks will likely adopt millimeter wave (mm-wave) and massive multiple-input-multiple-output (MIMO) technologies, due to a number of favorable properties. In particular, operating at carrier frequencies between 30 and $300 \mathrm{GHz}$, with large available bandwidths and with highly directional communication, mm-wave can provide extremely high data rates to users through dense spatial multiplexing by using a large number of antennas [1], [2]. While these properties are desirable for $5 \mathrm{G}$ services, $\mathrm{mm}$-wave communications also face a number of challenges. Among these challenges, the severe path loss at those high carrier frequencies stands out. The resulting loss in signal-to-noise ratio (SNR) must be countered through sophisticated beamforming at the transmitter and/or receiver side, leading to highly directional links [3]-[5]. However, this in turn requires knowledge of the propagation channel. Such knowledge is generally obtained through pilot signals, which in turn requires some initial alignment.

Location information can serve as a proxy for channel information to perform beamforming: when the location of the user is known, the base station can steer its transmission to the user, either directly or through a reflected path. This leads to a synergy between localization and communication. However, in order to reap the benefits of location information for highly directional communications, both the devices' positions and orientations should be accurately estimated. Today's technologies cannot always provide this information in an efficient and reliable manner. For instance, the global positioning system (GPS) is a well-established technology that can provide location information outdoors. However, in GPS-challenged environments (e.g., indoors and in urban canyons), the accuracy is poor. Other radio technologies, such as ultra-wide bandwidth (UWB) can be a solution for indoor positioning, but requires additional infrastructure and suffers

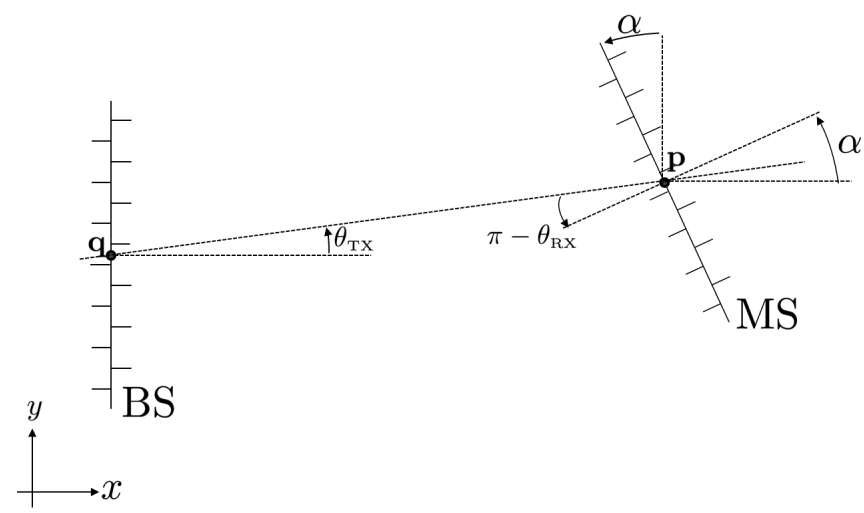

Fig. 1. Two dimensional illustration of the single-link based positioning problem. The BS location $\mathbf{q}$ and BS orientation are known, but arbitrary. The location of the MS $\mathbf{p}$, rotation angle $\alpha$, AOA $\theta_{\mathrm{RX}}$, AOD $\theta_{\mathrm{TX}}$, the channel between BS and MS, and the distance between the antenna centers are unknown.

from high hardware complexity [6].

In this paper, we show that mm-wave and massive MIMO, which are both candidate features for $5 \mathrm{G}$ communication networks, are also enabling technologies for accurate positioning and device orientation estimation. The limited scattering and high-directivity are unique characteristics of mm-wave channel and massive MIMO, respectively. In the line-of-sight (LOS) conditions, angle-of-departure (AOD) is used for the estimation of position while angle-of-arrival (AOA) provides the estimation of orientation. By exploiting these features together with beamforming, we provide sufficient conditions under which the Fisher information matrix (FIM) of the AOA, AOD and time-of-arrival (TOA) of the LOS path of a scenario illustrated in Fig. 1 is non-singular, in the presence of an unknown channel. A non-singular FIM is a necessary condition for the identifiability of the position and orientation of the user.

\section{RELATED WORK}

The use of 5G technologies to obtain position and orientation was previously explored in [7]-[9] for mm-wave and in [10]-[12] for massive MIMO. The early work [7] considered estimation and tracking of DOA through beam-switching. User localization was treated in [8], formulated as a hypothesis testing problem, limiting the spatial resolution. A different approach was taken in [9], where meter-level positioning 
accuracy was obtained by measuring received signal strength levels. In the massive MIMO case, [10] considered estimation of angles, while [11] treated joint estimation of delay, AOD, and $\mathrm{AOA}$, and evaluated the impact of errors in delays and phase shifters. However, no formal treatment regarding sufficient conditions for well-posed position and orientation estimation with one base station (BS) were provided. In [12], positioning was solved using a Gaussian process regressor, operating on a vector of received signal strengths through fingerprinting.

\section{SYSTEM MODEL}

We consider a MIMO system with a BS equipped with $N_{t}$ antennas and a mobile station (MS) equipped by $N_{r}$ antennas. Let $\mathbf{p}=\left[p_{x}, p_{y}\right]^{\mathrm{T}} \in \mathbb{R}^{2}$ and $\mathbf{q}=\left[q_{x}, q_{y}\right]^{\mathrm{T}} \in \mathbb{R}^{2}$ be locations of the MS and BS and let $\alpha \in[0,2 \pi)$ be the rotation angle of the MS's antenna array, with respect to the horizontal axis (see Fig. 1).

\section{A. Transmitter Model}

The transmitted signal is given by $\mathbf{F x}(t)$, where $\mathbf{F}=$ $\left[\mathbf{f}_{1}, \mathbf{f}_{2}, \ldots, \mathbf{f}_{M_{t}}\right] \in \mathbb{C}^{N_{t} \times M_{t}}$ is a beamforming matrix, in which $M_{t} \leq N_{t}$ denotes the number of beams. The signal transmitted ${ }^{1}$ over the $M_{t}$ beams is denoted by $\mathbf{x}(t)=$ $\left[x_{1}(t), \ldots, x_{M_{t}}(t)\right]^{\mathrm{T}} \in \mathbb{C}^{M_{t}}$.

\section{B. Channel Model}

In contrast to [11], we consider only line-of-sight (LOS) narrowband communication with a bandwidth $B$ and a carrier frequency $f_{c}$. In particular, the narrowband assumption involves (i) $B$ is sufficiently small such that the path loss can be considered constant over the signaling band; and (ii) the phase difference between upper and lower band edges for propagation across the entire array is small, i.e., $\max \left(N_{t}, N_{r}\right) d \ll c / B$ in which $d$ denotes the distance between the antenna elements and $c$ is the speed of light. Under these assumptions, the channel can be expressed as an $N_{r} \times N_{t}$ matrix

$$
\mathbf{H}=\sqrt{\frac{N_{t} N_{r}}{\rho}} h \mathbf{a}_{\mathrm{Rx}}\left(\theta_{\mathrm{Rx}}\right) \mathbf{a}_{\mathrm{Tx}}^{\mathrm{H}}\left(\theta_{\mathrm{Tx}}\right),
$$

in which $\rho$ is the path loss between BS and MS, $h$ is the complex gain of the LOS path, $\mathbf{a}_{\mathrm{Tx}}\left(\theta_{\mathrm{Tx}}\right) \in \mathbb{C}^{N_{t}}$ and $\mathbf{a}_{\mathrm{Rx}}\left(\theta_{\mathrm{Rx}}\right) \in \mathbb{C}^{N_{r}}$ are the antenna steering and response vectors, $\theta_{\mathrm{Tx}}$ and $\theta_{\mathrm{Rx}}$ are the AOD and AOA, respectively, as depicted in Fig. 1. For concreteness, we will consider a uniform linear array (ULA) at the transmitter, so that the vector $\mathbf{a}_{\mathrm{Tx}}\left(\theta_{\mathrm{Tx}}\right)$ can be written as

$$
\begin{aligned}
& \mathbf{a}_{\mathrm{Tx}}\left(\theta_{\mathrm{Tx}}\right)= \\
& \frac{1}{\sqrt{N_{t}}}\left[1, e^{j \frac{2 \pi}{\lambda} d \sin \left(\theta_{\mathrm{Tx}}\right)}, \ldots, e^{j\left(N_{t}-1\right) \frac{2 \pi}{\lambda} d \sin \left(\theta_{\mathrm{Tx}}\right)}\right]^{\mathrm{T}},
\end{aligned}
$$

\footnotetext{
${ }^{1}$ Unlike the system model in [13], where beams are sequentially transmitted one at the time in each observation time interval, we exploit beamforming to multiplexing data onto orthogonal spatial beams simultaneously, i.e., beamspace MIMO (B-MIMO) [8], [14], [15]. As a result, we obtain a signal that allows the estimation of AOA, AOD and TOA jointly. This improves the fundamental bounds on the estimation of position and orientation of the user significantly.
}

where $\lambda=c / f_{c}$ is the signal wavelength. Correspondingly, we will assume an ULA at the receiver, thus $\mathbf{a}_{\mathrm{Rx}}\left(\theta_{\mathrm{Rx}}\right)$ can be expressed similarly, replacing the subscript $\mathrm{Tx}$ by $\mathrm{Rx}$ in (2), and replacing $N_{t}$ by $N_{r}$. For notational convenience, we will use the notation $\mathbf{a}_{\mathrm{Tx}}$ instead of $\mathbf{a}_{\mathrm{Tx}}\left(\theta_{\mathrm{Tx}}\right)$. It should be noted that our analysis can be extended to any antenna configuration at transmitter and receiver, provided the above mentioned narrowband assumptions are met.

\section{Receiver Model}

The observed $N_{r} \times 1$ signal is given by

$$
\mathbf{y}(t)=\mathbf{H F} \mathbf{x}(t-\tau)+\mathbf{n}(t),
$$

where $\tau=\|\mathbf{q}-\mathbf{p}\| / c$ is the propagation delay between BS and MS and $\mathbf{n}(t) \in \mathbb{C}^{N_{r}}$ is a Gaussian noise vector with zero mean and two-side power spectral density $N_{0} / 2$.

Our goal is to determine position $\mathbf{p}$ and orientation $\alpha$ of the MS from $\mathbf{y}(t)$, observed during the time interval $t \in\left[0, T_{\mathrm{ob}}\right)$. Note that we do not assume any specific receiver-side processing or beamforming, as we aim to derive a fundamental lower bound.

\section{DERIVATION OF THE Fundamental BOUNDS}

In this section, we derive the FIM and the Cramér-Rao bound (CRB) for the estimation problem of the position and orientation of a MS using mm-wave MIMO. First, as an intermediate step, we construct the FIM of the channel parameters: delay, AOD, AOA, and the real and imaginary parts of the channel coefficients. Then, by means of a bijective transformation we obtain the FIM and thus the CRB of the position and rotation angle, which are the final parameters of interest.

\section{A. FIM: Channel Parameters}

Let $\boldsymbol{\eta} \in \mathbb{R}^{5}$ be the vector consisting of the unknown channel parameters (delay, AOD, AOA, and channel coefficients)

$$
\boldsymbol{\eta}=\left[\tau, \boldsymbol{\theta}^{\mathrm{T}}, \mathbf{h}^{\mathrm{T}}\right]^{\mathrm{T}},
$$

where $\mathbf{h}=\left[h_{\mathrm{R}}, h_{\mathrm{I}}\right]^{\mathrm{T}}$ with $h_{\mathrm{R}}$ and $h_{\mathrm{I}}$ being the real and imaginary parts of $h, \boldsymbol{\theta}=\left[\theta_{\mathrm{Tx}}, \theta_{\mathrm{Rx}}\right]^{\mathrm{T}}$.

Let $\hat{\boldsymbol{\eta}}$ be an unbiased estimator of $\boldsymbol{\eta}$. The mean squared error (MSE) of $\hat{\boldsymbol{\eta}}-\boldsymbol{\eta}$ is bounded as [16]

$$
\mathbb{E}_{\mathbf{y} \mid \boldsymbol{\eta}}\left[(\hat{\boldsymbol{\eta}}-\boldsymbol{\eta})(\hat{\boldsymbol{\eta}}-\boldsymbol{\eta})^{\mathrm{T}}\right] \succeq \mathbf{J}_{\boldsymbol{\eta}}^{-1}
$$

in which $\mathbb{E}_{\mathbf{y} \mid \boldsymbol{\eta}}[$.$] denotes the expectation parameterized by the$ unknown parameters $\boldsymbol{\eta}$, and $\mathbf{J}_{\boldsymbol{\eta}}$ is the $5 \times 5$ FIM defined as

$$
\mathbf{J}_{\boldsymbol{\eta}} \triangleq \mathbb{E}_{\mathbf{y} \mid \boldsymbol{\eta}}\left[-\frac{\partial^{2} \ln f(\mathbf{y} \mid \boldsymbol{\eta})}{\partial \boldsymbol{\eta} \partial \boldsymbol{\eta}^{T}}\right]
$$

where $f(\mathbf{y} \mid \boldsymbol{\eta})$ is the likelihood ratio of the random vector $\mathbf{y}$ obtained from the Karhunen-Loeve expansion of $\mathbf{y}(t)$ conditioned on $\boldsymbol{\eta}$. More specifically, $f(\mathbf{y} \mid \boldsymbol{\eta})$ can be written as [17]

$f(\mathbf{y} \mid \boldsymbol{\eta}) \propto \exp \left\{\frac{2}{N_{0}} \int_{0}^{T_{\mathrm{ob}}} \boldsymbol{\mu}^{\mathrm{H}}(t) \mathbf{y}(t) \mathrm{d} t-\frac{1}{N_{0}} \int_{0}^{T_{\mathrm{ob}}}\|\boldsymbol{\mu}(t)\|^{2} \mathrm{~d} t\right\}$, 
where $\boldsymbol{\mu}(t) \triangleq \mathbf{H F x}(t-\tau)$. The FIM in (6) can be structured as

$$
\mathbf{J}_{\boldsymbol{\eta}}=\left[\begin{array}{lll}
\Phi(\tau, \tau) & \Phi(\tau, \boldsymbol{\theta}) & \Phi(\tau, \mathbf{h}) \\
\Phi(\boldsymbol{\theta}, \tau) & \Phi(\boldsymbol{\theta}, \boldsymbol{\theta}) & \Phi(\boldsymbol{\theta}, \mathbf{h}) \\
\Phi(\mathbf{h}, \tau) & \Phi(\mathbf{h}, \boldsymbol{\theta}) & \Phi(\mathbf{h}, \mathbf{h})
\end{array}\right],
$$

in which the operator ${ }^{2} \Phi\left(\mathbf{x}_{1}, \mathbf{x}_{2}\right)$ is defined as

$$
\Phi\left(\mathbf{x}_{1}, \mathbf{x}_{2}\right) \triangleq \mathbb{E}_{\mathbf{y} \mid \boldsymbol{\eta}}\left[-\frac{\partial^{2} \ln f(\mathbf{y} \mid \boldsymbol{\eta})}{\partial \mathbf{x}_{1} \partial \mathbf{x}_{2}}\right] .
$$

The entries of the FIM are derived in Appendix A. Here we only summarize terms including $\tau$ and $\boldsymbol{\theta}$, taking into account that the norm of $\mathbf{a}_{\mathrm{Rx}}\left(\theta_{\mathrm{Rx}}\right)$ is one and hence it is omitted:

$$
\begin{aligned}
\Phi(\tau, \tau) & =\gamma|h|^{2} \mathbf{a}_{\mathrm{Tx}, \mathbf{F}}^{\mathrm{H}} \mathbf{A}_{2} \mathbf{a}_{\mathrm{Tx}, \mathbf{F}}, \\
\Phi\left(\tau, \theta_{\mathrm{Tx}}\right) & =\gamma|h|^{2} \Re\left\{j \mathbf{a}_{\mathrm{D}_{\mathrm{Tx}}, \mathbf{F}}^{\mathrm{H}} \mathbf{A}_{1} \mathbf{a}_{\mathrm{Tx}, \mathbf{F}}\right\}, \\
\Phi\left(\tau, \theta_{\mathrm{Rx}}\right) & =\gamma|h|^{2} \Re\left\{j A_{\mathbf{D}_{\mathrm{Rx}}} \mathbf{a}_{\mathrm{Tx}, \mathbf{F}}^{\mathrm{H}} \mathbf{A}_{1} \mathbf{a}_{\mathrm{Tx}, \mathbf{F}}\right\}, \\
\Phi\left(\theta_{\mathrm{Tx}}, \theta_{\mathrm{Tx}}\right) & =\gamma|h|^{2} \mathbf{a}_{\mathbf{D}_{\mathrm{Tx}}, \mathbf{F}} \mathbf{A}_{0} \mathbf{a}_{\mathbf{D}_{\mathrm{Tx}}, \mathbf{F}}, \\
\Phi\left(\theta_{\mathrm{Tx}}, \theta_{\mathrm{Rx}}\right) & =\gamma|h|^{2} \Re\left\{A_{\mathrm{D}_{\mathrm{Rx}}} \mathbf{a}_{\mathrm{Tx}, \mathbf{F}}^{\mathrm{H}} \mathbf{A}_{0} \mathbf{a}_{\mathbf{D}_{\mathrm{Tx}}, \mathbf{F}}\right\} \\
\Phi\left(\theta_{\mathrm{Rx}}, \theta_{\mathrm{Rx}}\right) & =\gamma|h|^{2} A_{\mathbf{D d}_{\mathrm{Rx}}} \mathbf{a}_{\mathrm{Tx}, \mathbf{F}}^{\mathrm{H}} \mathbf{A}_{0} \mathbf{a}_{\mathrm{Tx}, \mathbf{F}},
\end{aligned}
$$

where $\gamma=2 N_{t} N_{r} /\left(\rho N_{0}\right)$. We have introduced a number of notations: $\mathbf{A}_{n}, n \in\{0,1,2\}$, is given by

$$
\mathbf{A}_{n} \triangleq \int_{-B / 2}^{B / 2} \omega^{n} \mathbf{x}(\omega) \mathbf{x}^{\mathrm{H}}(\omega) \mathrm{d} \omega
$$

where the integral is applied element-wise, $\mathbf{x}(\omega)=$ $\left[x_{1}(\omega), \ldots, x_{M_{t}}(\omega)\right]^{\mathrm{T}}$ wherein $x_{i}(\omega)$ is the Fourier transform of $x_{i}(t)$. The vectors $\mathbf{a}_{\mathrm{Tx}, \mathbf{F}}$ and $\mathbf{a}_{\mathbf{D}_{\mathrm{Tx}}, \mathbf{F}}$ are given by $\mathbf{a}_{\mathrm{Tx}, \mathbf{F}}=\mathbf{F}^{\mathrm{H}} \mathbf{a}_{\mathrm{Tx}}, \mathbf{a}_{\mathbf{D}_{\mathrm{Tx}}, \mathbf{F}}=\mathbf{F}^{\mathrm{H}} \mathbf{D}_{\mathrm{Tx}} \mathbf{a}_{\mathrm{Tx}}$. The matrix $\mathbf{D}_{\mathrm{Tx}}$ is defined as

$$
\mathbf{D}_{\mathrm{Tx}} \triangleq j \frac{2 \pi}{\lambda} d \cos \left(\theta_{\mathrm{Tx}}\right) \operatorname{diag}\left\{0, \ldots, N_{\mathrm{Tx}}-1\right\} .
$$

The scalars $A_{\mathrm{D}_{\mathrm{Rx}}}$ and $A_{\mathrm{Dd}_{\mathrm{Rx}}}$ are defined as

$$
\begin{gathered}
A_{\mathbf{D}_{\mathrm{Rx}}} \triangleq \mathbf{a}_{\mathrm{Rx}}^{\mathrm{H}} \mathbf{D}_{\mathrm{Rx}} \mathbf{a}_{\mathrm{Rx}}, \\
A_{\mathbf{D d}_{\mathrm{Rx}}} \triangleq \mathbf{a}_{\mathrm{Rx}}^{\mathrm{H}} \mathbf{D}_{\mathrm{Rx}} \mathbf{D}_{\mathrm{Rx}}^{\mathrm{H}} \mathbf{a}_{\mathrm{Rx}},
\end{gathered}
$$

where $\mathbf{D}_{\mathrm{Rx}}$ has the same expression as (17) by replacing the subscript $\mathrm{Tx}$ by $\mathrm{Rx}$, and $\mathbf{a}_{\mathrm{Rx}}\left(\theta_{\mathrm{Rx}}\right)$ is replaced by $\mathbf{a}_{\mathrm{Rx}}$ for notational convenience.

\section{B. CRB for Position and Orientation}

In this section, we derive the FIM of the unknown position and orientation of the MS. We consider a parameter transformation from $\boldsymbol{\eta}$ to $\tilde{\boldsymbol{\eta}} \triangleq\left[\mathbf{p}^{\mathrm{T}}, \alpha, \mathbf{h}^{\mathrm{T}}\right]^{\mathrm{T}}$, where $\boldsymbol{\eta}$ and $\tilde{\boldsymbol{\eta}}$ are related by (see also Fig. 1): $\tau=\|\mathbf{q}-\mathbf{p}\| / c, \cos \left(\theta_{\mathrm{Tx}}\right)=$ $\left(p_{x}-q_{x}\right) /(\|\mathbf{q}-\mathbf{p}\|)$, and $\alpha=\pi+\theta_{\mathrm{Tx}}-\theta_{\mathrm{Rx}}$. This allows us to express $\boldsymbol{\eta}$ as a function of $\tilde{\boldsymbol{\eta}}$. Therefore, by means of a bijective transformation, the FIM of $\tilde{\boldsymbol{\eta}}$ can be obtained as

$$
\mathbf{J}_{\tilde{\eta}}=\mathbf{T} \mathbf{J}_{\eta} \mathbf{T}^{\mathrm{H}},
$$

where

$$
\mathbf{T}=\left[\begin{array}{lll}
\frac{\partial \tau}{\partial \mathbf{p}} & \frac{\partial \boldsymbol{\theta}^{\mathrm{T}}}{\partial \mathbf{p}} & \frac{\partial \mathbf{h}^{\mathrm{T}}}{\partial \mathbf{p}^{\mathrm{p}}} \\
\frac{\partial \tau}{\partial \alpha} & \frac{\partial \boldsymbol{\theta}^{\mathrm{T}}}{\partial \alpha} & \frac{\partial \mathbf{h}^{\mathrm{T}}}{\partial \alpha} \\
\frac{\partial \tau}{\partial \mathbf{h}} & \frac{\partial \boldsymbol{\theta}^{\mathrm{T}}}{\partial \mathbf{h}} & \frac{\partial \mathbf{h}^{\mathrm{T}}}{\partial \mathbf{h}}
\end{array}\right]
$$

\footnotetext{
${ }^{2}$ If $\mathbf{x}_{1}$ and $\mathbf{x}_{2}$ are scalars, then $\Phi\left(\mathbf{x}_{1}, \mathbf{x}_{2}\right)$ is also a scalar.
}

in which $\partial \tau / \partial \mathbf{p}=c / \mathbf{u}$,

$$
\frac{\partial \theta_{\mathrm{Tx}}}{\partial \mathbf{p}}=\frac{\partial \theta_{\mathrm{Rx}}}{\partial \mathbf{p}}=\frac{1}{\|\mathbf{q}-\mathbf{p}\|}\left[\begin{array}{cc}
0 & -1 \\
1 & 0
\end{array}\right] \mathbf{u}
$$

and $\partial \theta_{\mathrm{Rx}} / \partial \alpha=-1, \partial \mathbf{h}^{\mathrm{T}} / \partial \mathbf{h}=\mathbf{I}_{2}$ where $\mathbf{I}_{2}$ is the $2 \times 2$ identity matrix, and $\mathbf{u}=\left[\cos \left(\theta_{\mathrm{Tx}}\right), \sin \left(\theta_{\mathrm{Tx}}\right)\right]^{\mathrm{T}}$. The remaining terms in the transformation matrix $\mathbf{T}$ are zero. It can be seen that the transformation matrix $\mathbf{T}$ has full rank.

\section{Non-Singular FiM with One Base Station}

Now, we provide sufficient conditions for $\mathbf{J}_{\eta}$ to be nonsingular. Note that these conditions also imply that $\mathbf{J}_{\tilde{\eta}}$ is nonsingular, since $\mathbf{T}$ has full rank. For notational convenience we will limit ourselves to signals $\mathbf{x}(t)$ for which $\mathbf{A}_{1}=\mathbf{0}$. We also limit ourselves to well-posed systems, for which all diagonal elements in $\mathbf{J}_{\eta}$ are strictly greater than zero. This places certain natural requirements on the system (e.g., the number of transmit and receive antennas should be greater than 1), by ensuring that each parameter in $\boldsymbol{\eta}$ can be estimated when the other parameters are known.

Proposition 5.1: Consider a MIMO system, signal $\mathbf{x}(t)$, and beamforming matrix $\mathbf{F}$ designed such that the system is well-posed. For such as system $\mathbf{J}_{\eta}$ has full rank if and only if $\mathbf{A}_{0}$ has at least two non-zero eigenvalues.

Proof: See Appendix B.

From the proposition, it follows that it is required to use at least 2 beams and that the signal need to be properly designed in order to obtain a non-singular FIM. It should be noted that a non-singular FIM is in general not a sufficient condition for unambiguous determination of position and orientation, due to the possibility of multiple optimal points of the likelihood function.

\section{Simulation Results}

In this section, we present simulation results to demonstrate the performance of the proposed bounds with respect to different parameters.

\section{A. Simulation Setup}

We set $f_{c}=60 \mathrm{GHz}, B=100 \mathrm{MHz}, N_{0}=2 \mathrm{~W} / \mathrm{GHz}$ and $\rho=K\|\mathbf{q}-\mathbf{p}\|^{2}$ in which $K=1$. The inter-element spacing is assumed to be $d=\lambda / 2$. The number of transmit and receive antennas are set to $N_{t}=N_{r}=64$, while the number of beams is fixed to $M_{t}=3$. We have generated a signal $\mathbf{x}(t)$ for which $\mathbf{A}_{0}$ and $\mathbf{A}_{2}$ are $M_{t} \times M_{t}$ diagonal matrices ${ }^{3}$ and $\mathbf{A}_{1} \approx \mathbf{0}$. The system parameters are set such that within one meter of the transmitter, the SNR varies over a range of approximately 38 $\mathrm{dB}$. We consider a scenario that the BS is located at a fixed position $\mathbf{q}=[0,0]^{\mathrm{T}}$ and the MS is placed in a square area of $10 \mathrm{~m} \times 10 \mathrm{~m}$. We fix $\alpha=0 \mathrm{rad}$ and the unknown channel to $h=(1+j) / \sqrt{2}$. We use a $64 \times 3$ TX beamformer matrix $\mathbf{F}$ with the $i$-th column defined as

$$
\mathbf{f}_{i}=\left[1, e^{j \frac{2 \pi}{\lambda} d \sin \left(\psi_{i}\right)}, \ldots, e^{j\left(N_{t}-1\right) \frac{2 \pi}{\lambda} d \sin \left(\psi_{i}\right)}\right]^{\mathrm{T}},
$$




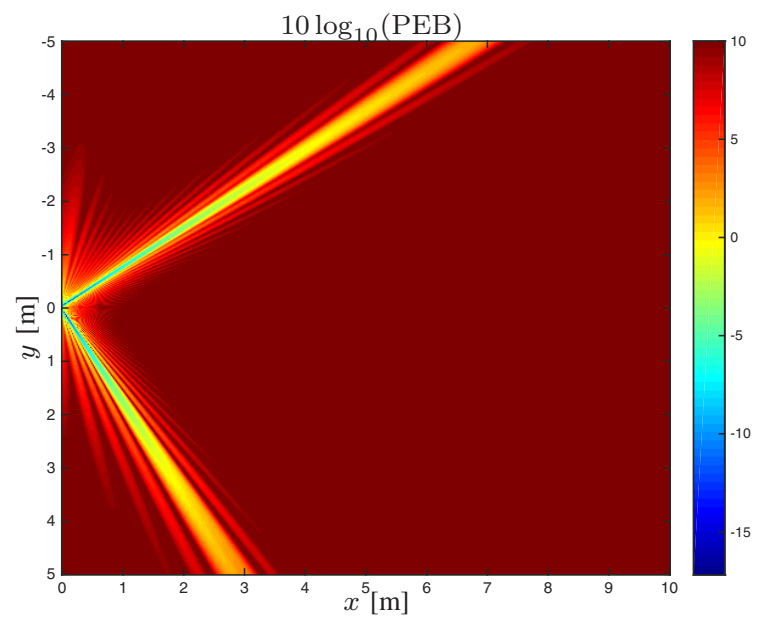

Fig. 2. PEB performance for different locations of the MS, for a scenario with 3 beams in the directions $[+\pi / 5,-\pi / 3,+\pi / 5+0.01]$. For visualization purposes, PEB values were truncated above $10 \mathrm{~m}$.

in which $\left[\psi_{1}, \psi_{2}, \psi_{3}\right]=[+\pi / 5,-\pi / 3,+\pi / 5+0.01]$.

Performance is measured in terms of the position error bound (PEB, expressed in meters) and the rotation error bound (REB, expressed in radians), where ${ }^{4} \mathrm{PEB} \triangleq$ $\sqrt{\operatorname{tr}\left\{\left[\mathbf{J}_{\tilde{\boldsymbol{\eta}}}^{-1}\right]_{1: 2,1: 2}\right\}}$, and REB $\triangleq \sqrt{\left[\mathbf{J}_{\tilde{\boldsymbol{\eta}}}^{-1}\right]_{3,3}}$.

\section{B. Results and Discussion}

Fig. 2 shows the PEB for different locations of the MS. As expected, in the directions of the beams, the PEB has the lowest values, while in the other locations the value of PEB is much higher. More specifically, for a distance of $\|\mathbf{q}-\mathbf{p}\|=1 \mathrm{~m}$, the PEB in the directions of the beams is approximately $17 \mathrm{~cm}$ (corresponding to $10 \log _{10}(\mathrm{PEB}) \approx 7.6$ ) while the highest $\mathrm{PEB}$ is approximately $13.7 \mathrm{~m}$ (corresponding to $10 \log _{10}(\mathrm{PEB}) \approx 11.4$ ), if we ignore values for $x \approx 0$. We note that while increasing the transmitted power will decrease the PEB, what is important to note from the figure is the extremely large range of PEB values, with variations over multiple orders of magnitude. We also observe the impact of the different beams: in the direction of $\pi / 5$, the two beams provide increased SNR, leading to better TOA information (in the Fisher sense) and good information regarding AOD. In the direction of $-\pi / 3$, the single beam provides good TOA information, but leads to poor AOD information (except for MS locations close to the BS). Hence, the PEB in the direction of $-\pi / 3$ is higher. At larger distances, say 5 meters, the PEB in the direction $\pi / 5$ is still around $86 \mathrm{~cm}$.

Fig. 3 shows REB for different locations of the MS. We observe that for the same conditions as in Fig. 2, the results confirm our previous findings. When the MS is within one meter of the BS, REB values range from $0.0014 \mathrm{rad}$ in the

\footnotetext{
${ }^{3}$ In particular $\mathbf{A}_{0} \approx 8 \times 10^{-3} \mathbf{I}$ and $\mathbf{A}_{2} \approx 6.6 \times 10^{-4} \mathbf{I}$, obtained by generating white Gaussian signals.

${ }^{4}$ Here $\left[\mathbf{J}_{\tilde{\eta}}^{-1}\right]_{1: 2,1: 2}$ denotes the $2 \times 2$ block from the first two rows and first two columns of the inverse of the FIM $\mathbf{J}_{\tilde{\eta}}$.
}

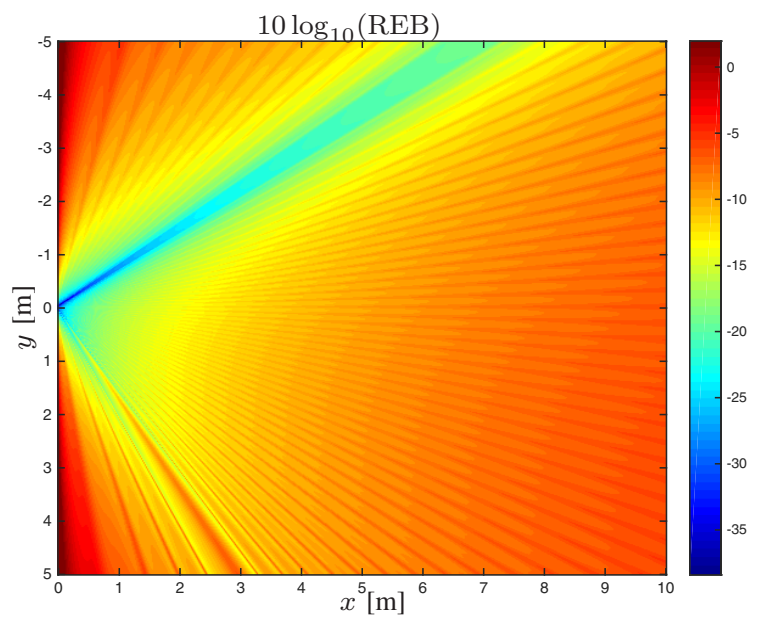

Fig. 3. REB performance for different locations of the MS, for a scenario with 3 beams in the directions $[+\pi / 5,-\pi / 3,+\pi / 5+0.01]$. For visualization purposes, REB values were truncated above $\pi / 2 \mathrm{rad}$.

direction $\pi / 5$, over $0.05 \mathrm{rad}$ in the direction $-\pi / 3$, up to $0.2 \mathrm{rad}$ outside any of the beams. We observe that in the direction $-\pi / 3$, the single beam cannot provide good REB values. This is because even though the AOA information is high, the low AOD information results in high REB in the direction $-\pi / 3$. Interestingly, even at larger distances, say 5 meters, REB values are still around $0.007 \mathrm{rad}$.

\section{CONCLUSiON}

We have presented a study of the fundamental bounds of a narrowband MIMO mm-wave system. Using a LOS channel model, we have first computed the FIM for delay, AOD, AOA, and channel gain. We have then transformed the FIM to comprise the position and rotation angle of MS. We have proved that obtaining a non-singular FIM with one BS in a LOS mm-wave system is possible by using multibeam transmission and appropriate signal design. Numerical results confirm that in the direction of isolated beams, neither position nor orientation can be estimated well, due to the poor information regarding in AOD. Multiple nearby beams provide good conditions for joint estimation of position and orientation.

\section{APPENDIX A}

\section{ELEMENTS IN (8)}

In what follows, we provide the proofs for the elements of the FIM presented in (10) to (15). Replacing $\mathbf{y}(t)$ from (3) in (7), using (9), and considering $\mathbb{E}_{\mathbf{y}}\left[\mathbf{n}_{Q}(t)\right]=\mathbf{0}$, we obtain

$$
\Phi\left(x_{1}, x_{2}\right)=\frac{2}{N_{0}} \int_{0}^{T_{\mathrm{ob}}} \Re\left\{\frac{\partial \boldsymbol{\mu}^{\mathrm{H}}(t)}{\partial x_{1}} \frac{\partial \boldsymbol{\mu}(t)}{\partial x_{2}}\right\} \mathrm{d} t .
$$

First of all,

$$
\begin{aligned}
\Phi(\tau, \tau) & =\frac{2}{N_{0}} \int_{0}^{T_{\mathrm{ob}}} \Re\left\{\frac{\partial \boldsymbol{\mu}^{\mathrm{H}}(t)}{\partial \tau} \frac{\partial \boldsymbol{\mu}(t)}{\partial \tau}\right\} \mathrm{d} t \\
& \stackrel{(a)}{=} \gamma|h|^{2} \int_{0}^{T_{\mathrm{ob}}} \Re\left\{\mathbf{a}_{\mathrm{Tx}, \mathbf{F}}^{\mathrm{H}} \mathbf{S}_{x}^{\prime \prime}(t-\tau) \mathbf{a}_{\mathrm{Tx}, \mathbf{F}}\right\} \mathrm{d} t,
\end{aligned}
$$


where (a) is the result of rearranging a complex scalar and defining $\mathbf{S}_{x}^{\prime \prime}(t-\tau)$ as

$$
\mathbf{S}_{x}^{\prime \prime}(t-\tau)=\frac{\partial \mathbf{x}(t-\tau)}{\partial \tau} \frac{\partial \mathbf{x}^{\mathrm{H}}(t-\tau)}{\partial \tau} .
$$

Finally, (10) is obtained by using the Parseval's identity.

The term $\Phi\left(\tau, \theta_{\mathrm{Tx}}\right)$ is obtained as follows:

$$
\begin{aligned}
& \Phi\left(\tau, \theta_{\mathrm{Tx}}\right)= \\
& \gamma|h|^{2} \int_{0}^{T_{\mathrm{ob}}} \Re\left\{\frac{\partial \mathbf{x}^{\mathrm{H}}(t-\tau)}{\partial \tau} \mathbf{a}_{\mathrm{Tx}, \mathbf{F}} \frac{\partial \mathbf{a}_{\mathrm{Tx}, \mathbf{F}}^{\mathrm{H}}}{\partial \theta_{\mathrm{Tx}}} \mathbf{x}(t-\tau)\right\} \mathrm{d} t .
\end{aligned}
$$

Since $\partial \mathbf{a}_{T x, \mathbf{F}}^{\mathrm{H}} / \partial \theta_{\mathrm{Tx}}=\mathbf{a}_{\mathrm{Tx}}^{\mathrm{H}} \mathbf{D}_{\mathrm{Tx}}^{\mathrm{H}} \mathbf{F}$, we find (11) after applying the Parseval's identity.

The term $\Phi\left(\tau, \theta_{\mathrm{Rx}}\right)$ is obtained as follows:

$\Phi\left(\tau, \theta_{\mathrm{Rx}}\right)=$

$\gamma|h|^{2} \int_{0}^{T_{\mathrm{ob}}} \Re\left\{\frac{\partial \mathbf{x}^{\mathrm{H}}(t-\tau)}{\partial \tau} \mathbf{a}_{\mathrm{Tx}, \mathbf{F}} \mathbf{a}_{\mathrm{Rx}}^{\mathrm{H}} \frac{\partial \mathbf{a}_{\mathrm{Rx}}}{\partial \theta_{\mathrm{Rx}}} \mathbf{a}_{\mathrm{Tx}, \mathbf{F}}^{\mathrm{H}} \mathbf{x}(t-\tau)\right\} \mathrm{d} t$.

Since $\partial \mathbf{a}_{\mathrm{Rx}} / \partial \theta_{\mathrm{Rx}}=\mathbf{D}_{\mathrm{Rx}} \mathbf{a}_{\mathrm{Rx}}$, applying the Parseval's identity leads to (12).

The term $\Phi(\tau, \mathbf{h})$ is obtained by taking the derivative with respect to real and imaginary parts of complex channel coefficient $h$ and applying the Parseval's identity, leading to

$$
\begin{aligned}
& \Phi(\tau, \mathbf{h})= \\
& \gamma\left[\Re\left\{j h^{*} \mathbf{a}_{\mathrm{Tx}, \mathbf{F}}^{\mathrm{H}} \mathbf{A}_{1} \mathbf{a}_{\mathrm{Tx}, \mathbf{F}}\right\},-\Re\left\{h^{*} \mathbf{a}_{\mathrm{T} \mathbf{x}, \mathbf{F}}^{\mathrm{H}} \mathbf{A}_{1} \mathbf{a}_{\mathrm{Tx}, \mathbf{F}}\right\}\right] .
\end{aligned}
$$

The term $\Phi\left(\theta_{\mathrm{Tx}}, \theta_{\mathrm{Tx}}\right)$ is obtained as

$$
\begin{aligned}
& \Phi\left(\theta_{\mathrm{Tx}}, \theta_{\mathrm{Tx}}\right)= \\
& \gamma|h|^{2} \int_{0}^{T_{\mathrm{ob}}} \Re\left\{\mathbf{x}^{\mathrm{H}}(t-\tau) \frac{\partial \mathbf{a}_{\mathrm{Tx}, \mathbf{F}}}{\partial \theta_{\mathrm{Tx}}} \frac{\partial \mathbf{a}_{\mathrm{Tx}, \mathbf{F}}^{\mathrm{H}}}{\partial \theta_{\mathrm{Tx}}} \mathbf{x}(t-\tau)\right\} \mathrm{d} t .
\end{aligned}
$$

Since $\partial \mathbf{a}_{\mathrm{Tx}, \mathbf{F}} / \partial \theta_{\mathrm{Tx}}=\mathbf{F}^{\mathrm{H}} \mathbf{D}_{\mathrm{Tx}} \mathbf{a}_{\mathrm{Tx}}$, we find (13).

The term $\Phi\left(\theta_{\mathrm{Tx}}, \theta_{\mathrm{Rx}}\right)$ is obtained as follows:

$\Phi\left(\theta_{\mathrm{Tx}}, \theta_{\mathrm{Rx}}\right)=$

$\gamma|h|^{2} \int_{0}^{T_{\mathrm{ob}}} \Re\left\{\mathbf{x}^{\mathrm{H}}(t-\tau) \frac{\partial \mathbf{a}_{\mathrm{Tx}, \mathbf{F}}}{\partial \theta_{\mathrm{Tx}}} \mathbf{a}_{\mathrm{Rx}}^{\mathrm{H}} \frac{\partial \mathbf{a}_{\mathrm{Rx}}}{\partial \theta_{\mathrm{Rx}}} \mathbf{a}_{\mathrm{Tx}, \mathbf{F}}^{\mathrm{H}} \mathbf{x}(t-\tau)\right\} \mathrm{d} t$.

Since $\partial \mathbf{a}_{\mathrm{Tx}, \mathbf{F}} / \partial \theta_{\mathrm{Tx}}=\mathbf{F}^{\mathrm{H}} \mathbf{D}_{\mathrm{Tx}} \mathbf{a}_{\mathrm{Tx}}$ and $\partial \mathbf{a}_{\mathrm{Rx}} / \partial \theta_{\mathrm{Rx}}=$ $\mathbf{D}_{\mathrm{Rx}} \mathbf{a}_{\mathrm{Rx}}$, applying the Parseval's identity leads to (14).

The term $\Phi\left(\theta_{\mathrm{Tx}}, \mathbf{h}\right)$ can be obtained by taking the derivatives with respect to real and imaginary parts of the channel coefficients and $\theta_{\mathrm{Tx}}$ and using the Parseval's identity as

$$
\begin{aligned}
& \Phi\left(\theta_{\mathrm{Tx}}, \mathbf{h}\right)= \\
& \gamma\left[\Re\left\{h^{*} \mathbf{a}_{\mathrm{Tx}, \mathbf{F}}^{\mathrm{H}} \mathbf{A}_{0} \mathbf{a}_{\mathbf{D}_{\mathrm{Tx}, \mathbf{F}}}\right\}, \Re\left\{j h^{*} \mathbf{a}_{\mathrm{Tx}, \mathbf{F}}^{\mathrm{H}} \mathbf{A}_{0} \mathbf{a}_{\mathbf{D}_{\mathrm{Tx}, \mathbf{F}}}\right\}\right] .
\end{aligned}
$$

The term $\Phi\left(\theta_{\mathrm{Rx}}, \theta_{\mathrm{Rx}}\right)$ is obtained as

$$
\begin{aligned}
& \Phi\left(\theta_{\mathrm{Rx}}, \theta_{\mathrm{Rx}}\right)= \\
& \gamma|h|^{2} \int_{0}^{T_{\mathrm{ob}}} \Re\left\{\mathbf{x}^{\mathrm{H}}(t-\tau) \mathbf{a}_{\mathrm{Tx}, \mathbf{F}} \frac{\partial \mathbf{a}_{\mathrm{Rx}}^{\mathrm{H}}}{\partial \theta_{\mathrm{Rx}}} \frac{\partial \mathbf{a}_{\mathrm{Rx}}}{\partial \theta_{\mathrm{Rx}}} \mathbf{a}_{\mathrm{Tx}, \mathbf{F}}^{\mathrm{H}} \mathbf{x}(t-\tau)\right\} \mathrm{d} t .
\end{aligned}
$$

Replacing the derivatives and using the Parseval's identity leads to (15).

The term $\Phi\left(\theta_{\mathrm{Rx}}, \mathbf{h}\right)$ can be obtained similarly by taking the first derivative with respect to $\theta_{\mathrm{Rx}}$ using the same procedure as (33), and the second derivative with respect to real and imaginary parts of channel coefficient $h$ and applying the Parseval's identity as

$\Phi\left(\theta_{\mathrm{Rx}}, \mathbf{h}\right)=$

$\gamma\left[\Re\left\{h^{*} A_{\mathbf{D}_{\mathrm{Rx}}}^{*} \mathbf{a}_{\mathrm{Tx}, \mathbf{F}}^{\mathrm{H}} \mathbf{A}_{0} \mathbf{a}_{\mathrm{Tx}, \mathbf{F}}\right\}, \Re\left\{j h^{*} A_{\mathbf{D}_{\mathrm{Rx}}}^{*} \mathbf{a}_{\mathrm{Tx}, \mathbf{F}}^{\mathrm{H}} \mathbf{A}_{0} \mathbf{a}_{\mathrm{Tx}, \mathbf{F}}\right\}\right]$.

Finally, the terms $\Phi\left(h_{\mathrm{R}}, h_{\mathrm{R}}\right), \Phi\left(h_{\mathrm{I}}, h_{\mathrm{I}}\right)$, and $\Phi\left(h_{\mathrm{R}}, h_{\mathrm{I}}\right)$ are obtained as

$$
\Phi\left(h_{\mathrm{R}}, h_{\mathrm{R}}\right)=\Phi\left(h_{\mathrm{I}}, h_{\mathrm{I}}\right)=\gamma \Re\left\{\mathbf{a}_{\mathrm{Tx}, \mathbf{F}}^{\mathrm{H}} \mathbf{A}_{0} \mathbf{a}_{\mathrm{Tx}, \mathbf{F}}\right\},
$$

and

$$
\Phi\left(h_{\mathrm{R}}, h_{\mathrm{I}}\right)=\gamma \Re\left\{j \mathbf{a}_{\mathrm{Tx}, \mathbf{F}}^{\mathrm{H}} \mathbf{A}_{0} \mathbf{a}_{\mathrm{Tx}, \mathbf{F}}\right\}=0,
$$

by taking the derivatives with respect to real and imaginary parts of channel coefficient $h$ and applying the Parseval's identity.

\section{APPENDIX B}

\section{PROOF FOR PROPOSITION 5.1}

The FIM of $\boldsymbol{\eta}$ is of the following form

$$
\mathbf{J}_{\boldsymbol{\eta}}=\left[\begin{array}{ccc}
\Phi(\tau, \tau) & \mathbf{0}^{\mathrm{T}} & \mathbf{0}^{\mathrm{T}} \\
\mathbf{0} & \boldsymbol{\Phi}(\boldsymbol{\theta}, \boldsymbol{\theta}) & \boldsymbol{\Phi}(\boldsymbol{\theta}, \mathbf{h}) \\
\mathbf{0} & \boldsymbol{\Phi}(\mathbf{h}, \boldsymbol{\theta}) & \boldsymbol{\Phi}(\mathbf{h}, \mathbf{h})
\end{array}\right]
$$

Due to the well-posed system requirement, $\Phi(\tau, \tau)>0$, so we only need to prove that the rank of $4 \times 4$ lower right matrix in (37) is 4 , since then $\mathbf{J}_{\boldsymbol{\eta}}$ would be full rank. We will denote this $4 \times 4$ matrix by $\mathbf{M}$.

We expand the positive semidefinite (PSD) matrix $\mathbf{A}_{0}$ as $\mathbf{A}_{0}=\mathbf{U} \boldsymbol{\Lambda} \mathbf{U}^{\mathrm{H}}$ in which $\mathbf{U}$ is the $M_{t} \times M_{t}$ matrix whose $i$ th column is the eigenvector $\mathbf{u}_{i}$ and $\boldsymbol{\Lambda}$ is a diagonal matrix whose diagonal elements are corresponding eigenvalues $\lambda_{i} \geq$ 0 . The eigenvalues are assumed to be ordered so that the first $\widetilde{M}_{t} \leq M_{t}$ eigenvalues are non-zero, while $\lambda_{i}=0$, for $i>\widetilde{M}_{t}$. Substituting $\mathbf{A}_{0}=\mathbf{U} \boldsymbol{\Lambda} \mathbf{U}^{\mathrm{H}}$ back into (37), and plugging in the appropriate entries from Appendix A, the lower $4 \times 4$ matrix can then be written as (without loss of generality, we set $\gamma=1$ ):

$$
\mathbf{M}=\sum_{i=1}^{\widetilde{M}_{t}} \lambda_{i}\left[\begin{array}{cc}
\zeta_{i} & \boldsymbol{\mu}_{i}^{\mathrm{T}} \\
\boldsymbol{\mu}_{i} & \boldsymbol{\Omega}_{i}
\end{array}\right]
$$

in which

$$
\begin{aligned}
& \zeta_{i}=|h|^{2}\left|\mathbf{a}_{\mathbf{D}_{\mathrm{Tx}}, \mathbf{F}}^{\mathrm{H}} \mathbf{u}_{i}\right|^{2}, \\
& \boldsymbol{\mu}_{i}=\left[|h|^{2} \Re\left\{A_{\mathbf{D}_{\mathrm{Rx}}} \beta_{i}\right\}, \Re\left\{h^{*} \beta_{i}\right\},-\Im\left\{h^{*} \beta_{i}\right\}\right]^{\mathrm{T}}, \\
& \boldsymbol{\Omega}_{i}=\left|\mathbf{a}_{\mathrm{Tx}, \mathbf{F}}^{\mathrm{H}} \mathbf{u}_{i}\right|^{2} \boldsymbol{\Omega},
\end{aligned}
$$

wherein $\beta_{i}=\mathbf{a}_{\mathrm{Tx}, \mathbf{F}}^{\mathrm{H}} \mathbf{u}_{i} \mathbf{u}_{i}^{\mathrm{H}} \mathbf{a}_{\mathbf{D}_{\mathrm{Tx}}, \mathbf{F}}$ and

$$
\boldsymbol{\Omega}=\left[\begin{array}{cc}
|h|^{2} A_{\mathbf{D d}} & \boldsymbol{\rho}^{\mathrm{T}} \\
\boldsymbol{\rho} & \mathbf{I}_{2}
\end{array}\right],
$$

in which $\boldsymbol{\rho}^{\mathrm{T}}=\left[\Re\left\{h^{*} A_{\mathbf{D}_{\mathrm{Rx}}}^{*}\right\},-\Im\left\{h^{*} A_{\mathbf{D}_{\mathrm{Rx}}}^{*}\right\}\right]$.

We introduce $\widetilde{\boldsymbol{\Omega}}=\sum_{i=1}^{M_{t}} \lambda_{i} \boldsymbol{\Omega}_{i}=\sum_{i=1}^{M_{t}} \lambda_{i}\left|a_{\mathrm{Tx}, \mathbf{F}}^{\mathrm{H}} \mathbf{u}_{i}\right|^{2} \boldsymbol{\Omega}$, $\tilde{\boldsymbol{\mu}}=\sum_{i=1}^{\widetilde{M}_{t}} \lambda_{i} \boldsymbol{\mu}_{i}$ and $\tilde{\zeta}=\sum_{i=1}^{\widetilde{M}_{t}} \lambda_{i} \zeta_{i}$. We recall that $\tilde{\zeta}>0$ due 
to the well-posed system. Regarding the lower $3 \times 3$ matrix of $\mathbf{M}$, we find that

$$
\begin{aligned}
\operatorname{det}(\widetilde{\boldsymbol{\Omega}}) & =\left(\sum_{i=1}^{\widetilde{M}_{t}} \lambda_{i}\left|\mathbf{a}_{\mathrm{Tx}, \mathbf{F}}^{\mathrm{H}} \mathbf{u}_{i}\right|^{2}\right)^{3} \operatorname{det}(\boldsymbol{\Omega}) \\
& =\left(\sum_{i=1}^{\widetilde{M}_{t}} \lambda_{i}\left|\mathbf{a}_{\mathrm{Tx}, \mathbf{F}}^{\mathrm{H}} \mathbf{u}_{i}\right|^{2}\right)^{3}|h|^{2}\left(A_{\mathbf{D d}_{\mathrm{Rx}}}-\left|A_{\mathbf{D}_{\mathrm{Rx}}}\right|^{2}\right)
\end{aligned}
$$

which is non-zero, since (i) $A_{\mathbf{D d}_{\mathrm{Rx}}}>\left|A_{\mathbf{D}_{\mathrm{Rx}}}\right|^{2}$ and (ii) if $\left|a_{\mathrm{Tx}, \mathbf{F}}^{\mathrm{H}} \mathbf{u}_{i}\right|^{2}=0, \forall i$, this would imply $\Phi\left(\theta_{\mathrm{Rx}}, \theta_{\mathrm{Rx}}\right)=0$, thus violating the well-posed system condition. Therefore, the rank of $\mathbf{M}$ is at least three.

Now, since

$$
\operatorname{det}(\mathbf{M})=\operatorname{det}(\widetilde{\boldsymbol{\Omega}})\left(\tilde{\zeta}-\tilde{\boldsymbol{\mu}}^{\mathrm{T}} \widetilde{\boldsymbol{\Omega}}^{-1} \tilde{\boldsymbol{\mu}}\right),
$$

the condition for $\mathbf{M}$ to be of full rank is that

$$
\tilde{\zeta}-\tilde{\boldsymbol{\mu}}^{\mathrm{T}} \widetilde{\boldsymbol{\Omega}}^{-1} \tilde{\boldsymbol{\mu}}>0 .
$$

Note that this condition is nothing else than the equivalent FIM of the AOD being strictly positive.

It is readily verified that

$$
\boldsymbol{\Omega}_{i}^{-1}=\frac{1}{\left|\mathbf{a}_{\mathrm{Tx}, \mathbf{F}}^{\mathrm{H}} \mathbf{u}_{i}\right|^{2} \operatorname{det}(\boldsymbol{\Omega})}\left[\begin{array}{cc}
1 & -\boldsymbol{\rho}^{\mathrm{T}} \\
-\boldsymbol{\rho} & \boldsymbol{\Psi}
\end{array}\right],
$$

where $\boldsymbol{\Psi}$ is the $2 \times 2$ matrix with elements

$$
\begin{aligned}
& \psi_{1,1}=|h|^{2} A_{\mathbf{D d}_{\mathrm{Rx}}}-\Im^{2}\left\{h^{*} A_{\mathbf{D}_{\mathrm{Rx}}}^{*}\right\}, \\
& \psi_{1,2}=-\Im\left\{h^{*} A_{\mathbf{D}_{\mathrm{Rx}}}^{*}\right\} \Re\left\{h^{*} A_{\mathbf{D}_{\mathrm{Rx}}}^{*}\right\}=\psi_{2,1}, \\
& \psi_{2,2}=|h|^{2} A_{\mathbf{D d}_{\mathrm{Rx}}}-\Re^{2}\left\{h^{*} A_{\mathbf{D}_{\mathrm{Rx}}}^{*}\right\} .
\end{aligned}
$$

Using the fact that

$$
\begin{aligned}
& |h|^{2} \Re\left\{A_{\mathbf{D}_{\mathrm{Rx}}} \beta_{i}\right\}=\Re\left\{\left(h^{*} \beta_{i}\right)\left(h^{*} A_{\mathbf{D}_{\mathrm{Rx}}}^{*}\right)^{*}\right\} \\
& =\Re\left\{h^{*} \beta_{i}\right\} \Re\left\{h^{*} A_{\mathbf{D}_{\mathrm{Rx}}}^{*}\right\}+\Im\left\{h^{*} \beta_{i}\right\} \Im\left\{h^{*} A_{\mathbf{D}_{\mathrm{Rx}}}^{*}\right\},
\end{aligned}
$$

we obtain

$$
\boldsymbol{\mu}_{i}^{\mathrm{T}} \boldsymbol{\Omega}_{i}^{-1} \boldsymbol{\mu}_{i}=\frac{|h|^{2}\left|\beta_{i}\right|^{2}}{\left|\mathbf{a}_{\mathrm{Tx}, \mathbf{F}}^{\mathrm{H}} \mathbf{u}_{i}\right|^{2}}=\zeta_{i},
$$

and

$$
\boldsymbol{\mu}_{i}^{\mathrm{T}} \boldsymbol{\Omega}^{-1} \boldsymbol{\mu}_{j}=|h|^{2} \Re\left\{\beta_{i} \beta_{j}^{*}\right\} .
$$

Substitution of $\tilde{\zeta}=\sum_{i=1}^{\widetilde{M}_{t}} \lambda_{i} \zeta_{i}$ and (51) and (52) into (45) yields the equivalent condition for full-rank $\mathbf{M}$ :

$$
\sum_{i=1}^{\widetilde{M}_{t}} \frac{\nu}{\nu_{i}}\left|\lambda_{i} \beta_{i}\right|^{2}-\left|\sum_{i=1}^{\widetilde{M}_{t}} \lambda_{i} \beta_{i}\right|^{2}>0,
$$

where $\nu_{i}=\lambda_{i}\left|\mathbf{a}_{\mathrm{Tx}, \mathbf{F}}^{\mathrm{H}} \mathbf{u}_{i}\right|^{2}, \nu=\sum_{i=1}^{\widetilde{M}_{t}} \nu_{i}$. We now distinguish two cases:

- Case $1\left(\widetilde{M}_{t}=1\right)$ : In this case $\nu_{i}=\nu$, and (53) is clearly not fulfilled. Hence, $\mathbf{M}$ does not have full rank and thus $\mathbf{J}_{\boldsymbol{\eta}}$ is not of full rank.

- Case $2\left(\widetilde{M}_{t}>1\right)$ : Now $\nu_{i}<\nu$, so that due to the CauchySchwarz inequality, (53) must be true. Hence, $\mathbf{M}$ and $\mathbf{J}_{\boldsymbol{\eta}}$ have full rank.

\section{ACKNOWLEDGMENT}

This work was supported by the European Commission, under EU FP7 Marie Curie Initial Training Network MULTIPOS (Multi-technology Positioning Professionals) under grant nr. 316528, the European Research Council, under grant nr. 258418 (COOPNET), the EU project HIGHTS (High precision positioning for cooperative ITS applications) MG3.5a-2014-636537, and the Spanish R+D project TEC2014$53656-\mathrm{R}$.

\section{REFERENCES}

[1] P. Zhouyue and F. Khan, "An introduction to millimeter-wave mobile broadband systems," IEEE Communications Magazine, vol. 49, no. 6, pp. 101-107, 2011.

[2] T. Rappaport, S. Sun, R. Mayzus, H. Zhao, Y. Azar, K. Wang, G. Wong, J. Schulz, M. Samimi, and F. Gutierrez, "Millimeter wave mobile communications for 5G cellular: It will work!" IEEE Access, vol. 1, pp. 335-349, 2013.

[3] J. Wang, "Beam codebook based beamforming protocol for multi-Gbps millimeter-wave WPAN systems," IEEE J. Sel. Areas Commun., vol. 27, no. 8, pp. 1390-1399, 2009.

[4] S. Hur, T. Kim, D. Love, J. Krogmeier, T. Thomas, and A. Ghosh, "Millimeter wave beamforming for wireless backhaul and accessin small cell networks," IEEE Trans. Commun., vol. 61, no. 10, pp. 4391-4403, 2013.

[5] Y. Tsang, A. Poon, and S. Addepalli, "Coding the beams: Improving beamforming training in mmwave communication system," in Global Telecomm. Conf. (GLOBECOM), 2011.

[6] Y. Shen and M. Z. Win, "Fundamental limits of wideband localization part I: A general framework," IEEE Transactions on Information Theory, vol. 56, no. 10, pp. 4956-4980, 2010.

[7] P. Sanchis, J. Martinez, J. Herrera, V. Polo, J. Corral, and J. Marti, "A novel simultaneous tracking and direction of arrival estimation algorithm for beam-switched base station antennas in millimeter-wave wireless broadband access networks," in IEEE Antennas and Propagation Society International Symposium, 2002.

[8] H. Deng and A. Sayeed, "Mm-wave MIMO channel modeling and user localization using sparse beamspace signatures," in International Workshop on Signal Processing Advances in Wireless Communications, 2014, pp. 130-134.

[9] M. Vari and D. Cassioli, "mmWaves RSSI indoor network localization," in ICC Workshop on Advances in Network Localization and Navigation, 2014.

[10] A. Hu, T. Lv, H. Gao, Z. Zhang, and S. Yang, "An ESPRIT-based approach for 2-D localization of incoherently distributed sources in massive MIMO systems," IEEE Journal of Selected Topics in Signal Processing, vol. 8, no. 5, pp. 996-1011, 2014.

[11] A. Guerra, F. Guidi, and D. Dardari, "Position and orientation error bound for wideband massive antenna arrays," in ICC Workshop on Advances in Network Localization and Navigation, 2015.

[12] V. Savic and E. G. Larsson, "Fingerprinting-based positioning in distributed massive MIMO systems," in IEEE Vehicular Technology Conference, 2015.

[13] A. Alkhateeb, O. E. Ayach, G. Leus, and R. W. Heath Jr, "Channel estimation and hybrid precoding for millimeter wave cellular systems," IEEE Journal of Selected Topics in Signal Processing, vol. 8, no. 5, pp. 831-846, 2014.

[14] J. Brady and A. Sayeed, "Beamspace MU-MIMO for high-density gigabit small cell access at millimeter-wave frequencies," in International Workshop on Signal Processing Advances in Wireless Communications, 2014, pp. 80-84.

[15] J. Brady, N. Behdad, and A. Sayeed, "Beamspace MIMO for millimeterwave communications: System architecture, modeling, analysis, and measurements," IEEE Transactions on Antennas and Propagation, vol. 61, no. 7, pp. 3814-3827, 2013.

[16] S. M. Kay, Fundamentals of Statistical Signal Processing: Estimation Theory. New York, NY, USA: Prentice Hall, 2010.

[17] H. V. Poor, An Introduction to Signal Detection and Estimation, 2nd ed. New York: Springer-Verlag, 1994. 